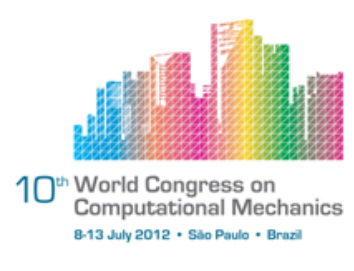

\title{
Topological derivatives for thermo-mechanical semi-coupled system
}

\author{
J.E. Esparta ${ }^{1}$ and S.M. Giusti ${ }^{2}$ \\ ${ }^{1}$ Laboratório Nacional de Computação Científica, LNCC/MCT. Av. Getúlio Vargas 333, \\ 25651-075 Petrópolis - RJ, Brasil \\ ${ }^{2}$ Universidad Tecnológica Nacional, Facultad Regional Córdoba UNT/FRC - CONICET, \\ Maestro M. López esq. Cruz Roja Argentina, X5016ZAA - Córdoba, Argentina \\ (sgiusti@ civil.frc.utn.edu.ar)
}

\begin{abstract}
The topological derivative measures the sensitivity of a given shape functional with respect to an infinitesimal singular domain perturbation. According to the literature, the topological derivative has been fully developed for a wide range of one single physical phenomenon modeled by partial differential equations. In addition, up to our knowledge, the topological asymptotic analysis associated to multi-physics problems has so far not been reported in the literature. In this work, we present the topological asymptotic analysis for the total potential mechanical energy associated to a thermo-mechanical system, when a small circular inclusion is introduced at an arbitrary point of the domain. In particular, we consider the linear elasticity system (modeled by the Navier equation) coupled with the steadystate heat conduction problem (modeled by the Laplace equation). The mechanical coupling term comes out from the thermal stress induced by the temperature field. Since this term is non-local, we introduce a non-standard adjoint state, which allows to obtain a closed form for the topological derivative. Finally, we provide a full mathematical justification for the derived formulas and develop precise estimates for the remainders of the topological asymptotic expansion.
\end{abstract}

Keywords: Topological derivative, thermo-mechanical system, multi-physic topology optimization, asymptotic analysis.

\section{Introduction}

The topological derivative measures the sensitivity of a given shape functional with respect to an infinitesimal singular domain perturbation, such as the insertion of holes, inclusions, source-terms or even cracks ([7]). The topological derivative was rigorously introduced by [19]. Since then, this concept has proved to be extremely useful in the treatment of a wide range of problems, for instance, topology optimization ([5, 18]), inverse analysis $([4,14])$ and image processing $([13,15])$, and has became a subject of intensive research. See, for instance, applications of the topological derivative in the multi-scale constitutive modeling 

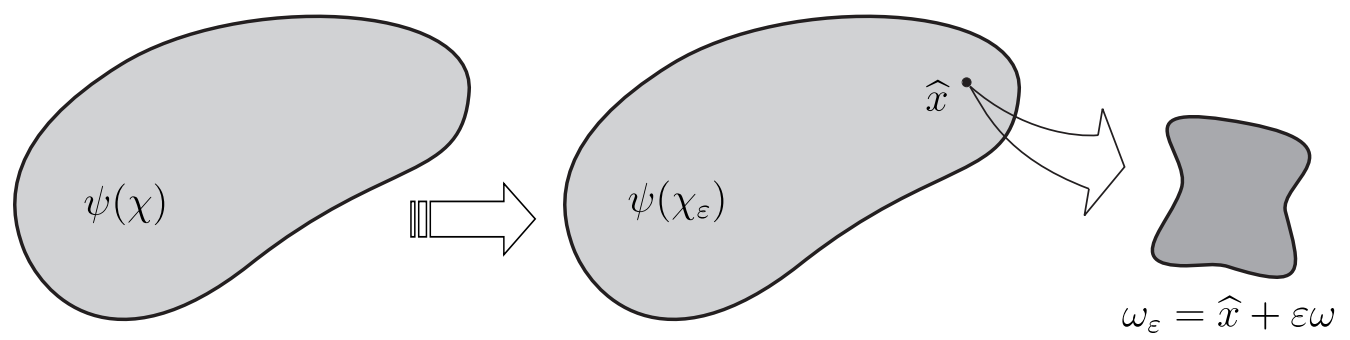

Figure 1. The topological derivative concept.

context $([3,9])$, fracture mechanics sensitivity analysis ([10]) and damage evolution modeling ([1]). Concerning the theoretical development of the topological asymptotic analysis, the reader may refer to the papers by [2] and [16], for instance.

In order to introduce these concepts, let us consider a bounded domain $\Omega \subset \mathbb{R}^{2}$, which is subject to a non-smooth perturbation confined in a small region $\omega_{\varepsilon}(\widehat{x})=\widehat{x}+\varepsilon \omega$ of size $\varepsilon$, as shown in fig. 1. Here, $\widehat{x}$ is an arbitrary point of $\Omega$ and $\omega$ is a fixed domain of $\mathbb{R}^{2}$. We introduce a characteristic function $x \mapsto \chi(x), x \in \mathbb{R}^{2}$, associated to the unperturbed domain, namely $\chi=\mathbb{1}_{\Omega}$. Then, we define a characteristic function associated to the topologically perturbed domain of the form $x \mapsto \chi_{\varepsilon}(\widehat{x} ; x), x \in \mathbb{R}^{2}$. In the case of a perforation, for instance, $\chi_{\varepsilon}(\widehat{x})=\mathbb{1}_{\Omega}-\mathbb{1}_{\overline{\omega_{\varepsilon}(\widehat{x})}}$ and the perforated domain is obtained as $\Omega_{\varepsilon}=\Omega \backslash \overline{\omega_{\varepsilon}}$. Then, we assume that a given shape functional $\psi\left(\chi_{\varepsilon}(\widehat{x})\right)$, associated to the topologically perturbed domain, admits the following topological asymptotic expansion

$$
\psi\left(\chi_{\varepsilon}(\widehat{x})\right)=\psi(\chi)+f(\varepsilon) D_{T} \psi(\widehat{x})+o(f(\varepsilon)),
$$

where $\psi(\chi)$ is the shape functional associated to the original (unperturbed) domain, $f(\varepsilon)$ is a positive function such that $f(\varepsilon) \rightarrow 0$, when $\varepsilon \rightarrow 0$. The function $\widehat{x} \mapsto D_{T} \psi(\widehat{x})$ is called the topological derivative of $\psi$ at $\widehat{x}$. Therefore, this derivative can be seen as a first order correction of $\psi(\chi)$ to approximate $\psi\left(\chi_{\varepsilon}(\widehat{x})\right)$. In fact, after rearranging (1) we have

$$
\frac{\psi\left(\chi_{\varepsilon}(\widehat{x})\right)-\psi(\chi)}{f(\varepsilon)}=D_{T} \psi(\widehat{x})+\frac{o(f(\varepsilon))}{f(\varepsilon)} .
$$

The limit passage $\varepsilon \rightarrow 0$ in the above expression leads to

$$
D_{T} \psi(\widehat{x})=\lim _{\varepsilon \rightarrow 0} \frac{\psi\left(\chi_{\varepsilon}(\widehat{x})\right)-\psi(\chi)}{f(\varepsilon)} .
$$

Since we are dealing with singular domain perturbations, the shape functionals $\psi\left(\chi_{\varepsilon}(\widehat{x})\right)$ and $\psi(\chi)$ are associated to topologically different domains. Therefore, the above limit is not trivial to be calculated. In particular, we need to perform an asymptotic analysis of the shape functional $\psi\left(\chi_{\varepsilon}(\widehat{x})\right)$ with respect to the small parameter $\varepsilon$. In order to calculate the topological derivative, in this work we will apply the methodology developed in [17]. The method is based on the following result:

$$
D_{T} \psi(\widehat{x})=\lim _{\varepsilon \rightarrow 0} \frac{1}{f^{\prime}(\varepsilon)} \frac{d}{d \varepsilon} \psi\left(\chi_{\varepsilon}(\widehat{x})\right) .
$$

The derivative of $\psi\left(\chi_{\varepsilon}(\widehat{x})\right)$ with respect to $\varepsilon$ can be seen as the sensitivity of $\psi\left(\chi_{\varepsilon}(\widehat{x})\right)$, in the classical sense $[6,20]$, to the domain variation produced by an uniform expansion of 
the perturbation $\omega_{\varepsilon}$, namely, $\omega_{\varepsilon+t}(\widehat{x})=\omega_{\varepsilon}(\widehat{x})+t \omega$. In fact, we have

$$
\frac{d}{d \varepsilon} \psi\left(\chi_{\varepsilon}(\widehat{x})\right)=\lim _{t \rightarrow 0} \frac{\psi\left(\chi_{\varepsilon+t}(\widehat{x})\right)-\psi\left(\chi_{\varepsilon}(\widehat{x})\right)}{t}
$$

where $\psi\left(\chi_{\varepsilon+t}(\widehat{x})\right)$ is the shape functional associated to the perturbed domain, whose perturbation is given by $\omega_{\varepsilon+t}$. Therefore, since $\psi\left(\chi_{\varepsilon+t}(\widehat{x})\right)$ and $\psi\left(\chi_{\varepsilon}(\widehat{x})\right)$ are now associated to topologically identical domains, we can use the concept of shape sensitivity analysis as an intermediate step in the topological derivative calculation. We will see later that this procedure enormously simplifies the analysis.

According to the literature, the topological derivative has been fully developed for a wide range of one single physical phenomenon modeled by partial differential equations. In addition, up to our knowledge, the topological asymptotic analysis associated to multi-physics problems has so far not been reported in the literature. In this work, therefore, we derive the topological derivative in its closed form for the total potential mechanical energy associated to a thermo-mechanical semi-coupled system, when a small circular inclusion is introduced at an arbitrary point of the domain. In particular, we consider the linear elasticity system (modeled by the Navier equation) coupled with the steady-state heat conduction problem (modeled by the Laplace equation). The mechanical coupling term comes out from the thermal stress induced by the temperature field. Since this term is non-local, we introduce a non-standard adjoint state, which simplifies the analysis allowing to obtain a closed form for the topological derivative. Finally, we provide a full mathematical justification for the derived formula and develop precise estimates for the remainders of the topological asymptotic expansion. We note that this result can be applied in technological research areas such as multi-physic topology design of structures under mechanical and/or thermal loads.

This paper is organized as follows. Section 2 describes the model associated to a thermo-mechanical semi-coupled problem. The topological sensitivity analysis is presented in Section 3, where the main result of this work - the topological derivative in its closed form for the total potential mechanical energy associated to a thermo-mechanical semi-coupled system - is derived. The paper ends in Section 4 where concluding remarks are presented.

\section{Formulation of the problem}

As mentioned in previous section, in this work the topological derivative of the total potential energy associated to the mechanical problem submitted to thermal stresses is derived. As topological perturbation we consider a nucleation of a small circular inclusion, with a contrast in the elastic, thermal and thermal-expansion constitutive properties. Then, it is needed to formulate the problems associated to the original and topological perturbed domains.

\subsection{Unperturbed problem}

Consider an open and bounded domain $\Omega \in \mathbb{R}^{2}$ representing an elastic solid body subject to a linear thermomechanical deformation process. Assuming small deformation and 
variations of temperatures, the functional that represents the total potential energy of the mechanical system is written as:

$$
\mathcal{J}_{\chi}(u, \theta):=\frac{1}{2} \int_{\Omega} \sigma(u) \cdot \nabla u^{s}-\int_{\Omega} Q(\theta) \cdot \nabla u^{s}-\int_{\Gamma_{N_{M}}} \bar{t} \cdot u,
$$

where $u$ represents the displacement field and $\bar{t}$ is a external traction acting on boundary $\Gamma_{N_{M}}$. The displacement field on the boundary $\Gamma_{D_{M}}$ satisfies $\left.u\right|_{\Gamma_{D_{M}}}=\bar{u}$, being $\bar{u}$ a prescribed displacement. Moreover, note that $\Gamma_{D_{M}} \cap \Gamma_{N_{M}}=\varnothing$ and $\overline{\Gamma_{D_{M}} \cup \Gamma_{N_{M}}}=\partial \Omega$. The Cauchy stress tensor $\sigma(u)$ in (6) is defined as:

$$
\sigma(u):=\mathbb{C} \nabla u^{s}
$$

where $\nabla u^{s}$ is used to denote the symmetric part of the gradient of the displacement field $u$, i.e.

$$
\nabla u^{s}:=\frac{1}{2}\left(\nabla u+(\nabla u)^{\top}\right) .
$$

The induced thermal stress tensor $Q(\theta)$ in (6) is defined as:

$$
Q(\theta):=\mathbb{C} B \theta
$$

where $\theta$ is the temperature field. In addition, $\mathbb{C}$ denotes the four-order elastic tensor and $B$ denotes the second-order thermo-elastic tensor. In the case of isotropic elastic body, theses tensors are given by:

$$
\mathbb{C}=2 \mu I I+\lambda(I \otimes I) \text { and } B=\alpha I \Rightarrow \mathbb{C} B=2 \alpha(\lambda+\mu) I
$$

with $\mu$ and $\lambda$ denoting the Lame's coefficients, and $\alpha$ the thermal expansion coefficient. In terms of the enginnering constant $E$ (Young's modulus) and $\nu$ (Poisson's ratio) the above constitutive response can be written as:

$$
\mathbb{C}=\frac{E}{1-\nu^{2}}[(1-\nu) I I+\nu(I \otimes I)] \text { and } \mathbb{C} B=\frac{\alpha E}{1-\nu} I .
$$

In addition, the field $u$ is the solution of the following variational problem: find $u \in$ $\mathcal{U}^{M}$, such that

$$
\int_{\Omega} S(u, \theta) \cdot \nabla \eta^{s}=\int_{\Gamma_{N_{M}}} \bar{t} \cdot \eta \quad \forall \eta \in \mathcal{V}^{M},
$$

with the tensor $S(u, \theta)$ representing the total stress, i.e. the contribution of the mechanical and thermal stresses,

$$
S(u, \theta)=\sigma(u)-Q(\theta) .
$$

In the variational problem (12), the set $\mathcal{U}^{M}$ and the space $\mathcal{V}^{M}$ are defined as $\mathcal{U}^{M}:=\left\{\phi \in H^{1}\left(\Omega ; \mathbb{R}^{2}\right): \phi=\bar{u}\right.$ on $\left.\Gamma_{D_{M}}\right\} \quad$ and $\mathcal{V}^{M}:=\left\{\phi \in H^{1}\left(\Omega ; \mathbb{R}^{2}\right): \phi=0\right.$ on $\left.\Gamma_{D_{M}}\right\}$.

Finally, the temperature field of the body $\theta$ is solution of the following variational problem: find $\theta \in \mathcal{U}^{T}$, such that

$$
\int_{\Omega} q(\theta) \cdot \nabla \eta=\int_{\Gamma_{N_{T}}} \bar{q} \eta \forall \eta \in \mathcal{V}^{T}
$$


where $\bar{q}$ is a prescribed heat flux on the Neumann boundary $\Gamma_{N_{T}}$. In the Dirichlet boundary $\Gamma_{D_{T}}$ there is a prescribed temperature denoted as $\bar{\theta}$. Then, $\Gamma_{D_{T}} \cap \Gamma_{N_{T}}=\varnothing$ and $\overline{\Gamma_{D_{T}} \cup \Gamma_{N_{T}}}=$ $\partial \Omega$. The heat flux operator $q(\theta)$ is defined as

$$
q(\theta)=-K \nabla \theta
$$

where $K$ is an second order tensor representing the thermal conductivity of the medium. In the isotropic case, the tensor $K$ can be written as

$$
K=k I
$$

being $k$ the thermal conductivity coefficient. In the variational problem (15), the set $\mathcal{U}^{T}$ and the space $\mathcal{V}^{T}$ are defined as:

$$
\mathcal{U}^{T}:=\left\{\phi \in H^{1}(\Omega): \phi=\bar{\theta} \text { on } \Gamma_{D_{T}}\right\} \quad \text { and } \mathcal{V}^{T}:=\left\{\phi \in H^{1}(\Omega): \phi=0 \text { on } \Gamma_{D_{T}}\right\} .
$$

In order to simplify further analysis, we introduce the following auxiliary problem: find $\varphi \in \mathcal{V}^{T}$, such that:

$$
\int_{\Omega} q(\varphi) \cdot \nabla \eta=\int_{\Omega} Q(\eta) \cdot \nabla u^{s} \quad \forall \eta \in \mathcal{V}^{T}
$$

\subsection{Perturbed problem}

Considering the introduction of a circular inclusion, denoted as $\omega_{\varepsilon}(\widehat{x}):=B_{\varepsilon}(\widehat{x})$, with radius $\varepsilon$ and centered at point $\widehat{x}$ in $\Omega$, the total potential energy functional associated to the perturbed domain mechanical system can be written as:

$$
\mathcal{J}_{\chi_{\varepsilon}}\left(u_{\varepsilon}, \theta_{\varepsilon}\right):=\frac{1}{2} \int_{\Omega} \sigma_{\varepsilon}\left(u_{\varepsilon}\right) \cdot \nabla u_{\varepsilon}^{s}-\int_{\Omega} Q_{\varepsilon}\left(\theta_{\varepsilon}\right) \cdot \nabla u_{\varepsilon}^{s}-\int_{\Gamma_{N_{M}}} \bar{t} \cdot u_{\varepsilon}
$$

where $u_{\varepsilon}$ and $\theta_{\varepsilon}$ denotes, respectively, the displacement and temperature fields, both associated to the perturbed system. In addition, $\sigma_{\varepsilon}\left(u_{\varepsilon}\right)$ and $Q_{\varepsilon}\left(\theta_{\varepsilon}\right)$ are used to denote the mechanical and the induced thermal stresses tensors associated to the perturbed problem. These tensors are defined as:

$$
\sigma_{\varepsilon}\left(u_{\varepsilon}\right):=\gamma_{\varepsilon}^{M} \mathbb{C} \nabla u_{\varepsilon}^{s} \text { and } Q_{\varepsilon}\left(\theta_{\varepsilon}\right):=\gamma_{\varepsilon}^{M} \gamma_{\varepsilon}^{C} \mathbb{C} B \theta_{\varepsilon},
$$

being $\gamma_{\varepsilon}^{M}$ and $\gamma_{\varepsilon}^{C}$ the contrast parameters in the constitutive properties, defined as

$$
\gamma_{\varepsilon}^{M}:=\left\{\begin{array}{cll}
1 & \text { in } & \Omega \backslash \overline{B_{\varepsilon}} \\
\gamma^{M} & \text { in } & B_{\varepsilon}
\end{array} \quad \text { and } \gamma_{\varepsilon}^{C}:=\left\{\begin{array}{cll}
1 & \text { in } & \Omega \backslash \overline{B_{\varepsilon}} \\
\gamma^{C} & \text { in } & B_{\varepsilon}
\end{array} .\right.\right.
$$

with $\gamma^{M}$ and $\gamma^{C}$ used to denote the values of the contrast. In the perturbed configuration, the displacement field satisfies the variational problem: find $u_{\varepsilon} \in \mathcal{U}_{\varepsilon}^{M}$, such that

$$
\int_{\Omega} S_{\varepsilon}\left(u_{\varepsilon}, \theta_{\varepsilon}\right) \cdot \nabla \eta^{s}=\int_{\Gamma_{N_{M}}} \bar{t} \cdot \eta \quad \forall \eta \in \mathcal{V}_{\varepsilon}^{M}
$$

where the total stress operator $S_{\varepsilon}\left(u_{\varepsilon}, \theta_{\varepsilon}\right)$ associated to the perturbed domain is given by

$$
S_{\varepsilon}\left(u_{\varepsilon}, \theta_{\varepsilon}\right)=\sigma_{\varepsilon}\left(u_{\varepsilon}\right)-Q_{\varepsilon}\left(\theta_{\varepsilon}\right) .
$$


The set $\mathcal{U}_{\varepsilon}^{M}$ and the space $\mathcal{V}_{\varepsilon}^{M}$ in the variational problem (23) are defined as

$$
\mathcal{U}_{\varepsilon}^{M}:=\left\{\phi \in \mathcal{U}^{M}: \llbracket \phi \rrbracket=0 \text { on } \partial B_{\varepsilon}\right\} \quad \text { and } \quad \mathcal{V}_{\varepsilon}^{M}:=\left\{\phi \in \mathcal{V}^{M}: \llbracket \phi \rrbracket=0 \text { on } \partial B_{\varepsilon}\right\}
$$

where the operator $\llbracket(\cdot) \rrbracket$ is introduced to denote the jump of $(\cdot)$ across the boundary of the perturbation.

In addition, the thermal equilibrium problem can be written in the variational form as: find $\theta_{\varepsilon} \in \mathcal{U}_{\varepsilon}^{T}$, such that

$$
\int_{\Omega} q_{\varepsilon}\left(\theta_{\varepsilon}\right) \cdot \nabla \eta=\int_{\Gamma_{N_{T}}} \bar{q} \eta \quad \forall \eta \in \mathcal{V}_{\varepsilon}^{T},
$$

with the thermal flux in the perturbed domain being defined as:

$$
q_{\varepsilon}\left(\theta_{\varepsilon}\right):=-\gamma_{\varepsilon}^{T} K \nabla \theta_{\varepsilon}
$$

where $\gamma_{\varepsilon}^{T}$ is the parameter that define the contrast between the thermal (constitutive) properties of the matrix and the inclusion, and is defined by:

$$
\gamma_{\varepsilon}^{T}:=\left\{\begin{array}{cll}
1 & \text { in } & \Omega \backslash \overline{B_{\varepsilon}} \\
\gamma^{T} & \text { in } & B_{\varepsilon}
\end{array}\right.
$$

being $\gamma^{T}$ the value of the contrast. In the variational problem (26) the set $\mathcal{U}_{\varepsilon}^{T}$ and the space $\mathcal{V}_{\varepsilon}^{T}$ are defined as:

$$
\mathcal{U}_{\varepsilon}^{T}:=\left\{\phi \in \mathcal{U}^{T}: \llbracket \phi \rrbracket=0 \text { on } \partial B_{\varepsilon}\right\} \quad \text { and } \quad \mathcal{V}_{\varepsilon}^{T}:=\left\{\phi \in \mathcal{V}^{T}: \llbracket \phi \rrbracket=0 \text { on } \partial B_{\varepsilon}\right\}
$$

Finally, the auxiliary problem (19), associated to the topologically perturbed domain is written as: find $\varphi_{\varepsilon} \in \mathcal{V}_{\varepsilon}^{T}$, such that:

$$
\int_{\Omega} q_{\varepsilon}\left(\varphi_{\varepsilon}\right) \cdot \nabla \eta=\int_{\Omega} Q_{\varepsilon}(\eta) \cdot \nabla u^{s} \quad \forall \eta \in \mathcal{V}_{\varepsilon}^{T}
$$

\section{Topological Sensitivity Analysis}

In order to proceed, it is convenient to introduce an analogy to classical continuum mechanics [11] where by the shape change velocity field $V$ is identified with the classical velocity field of a deforming continuum and $\varepsilon$ is identified as a time parameter. Since we are dealing with an uniform expansion of the inclusion $B_{\varepsilon}$, the shape velocity field $V$ satisfies: $\left.V\right|_{\partial \Omega}=0$ and $\left.V\right|_{\partial B_{\varepsilon}}=-n$. Then, the shape derivative of the functional (20) can be written as:

$$
\begin{aligned}
\dot{\mathcal{J}}_{\chi_{\varepsilon}}\left(u_{\varepsilon}, \theta_{\varepsilon}\right) & =\left(\frac{1}{2} \int_{\Omega} \sigma_{\varepsilon}\left(u_{\varepsilon}\right) \cdot \nabla u_{\varepsilon}^{s}-\int_{\Omega} Q_{\varepsilon}\left(\theta_{\varepsilon}\right) \cdot \nabla u_{\varepsilon}^{s}-\int_{\Gamma_{N_{M}}} \bar{t} \cdot u_{\varepsilon}\right) \\
& =\frac{1}{2}\left(\int_{\Omega} \sigma_{\varepsilon}\left(u_{\varepsilon}\right) \cdot \nabla u_{\varepsilon}^{s}\right)-\left(\int_{\Omega} Q_{\varepsilon}\left(\theta_{\varepsilon}\right) \cdot \nabla u_{\varepsilon}^{s}\right)-\int_{\Gamma_{N_{M}}} \bar{t} \cdot \dot{u}_{\varepsilon}
\end{aligned}
$$

where each term represents the derivative with respect to the parameter $\varepsilon$. Therefore, we can state the following propositions: 
Proposition 1 Let $\mathcal{J}_{\chi_{\varepsilon}}\left(u_{\varepsilon}, \theta_{\varepsilon}\right)$ be the functional defined by (20). Then, its derivative with respect to the small parameter $\varepsilon$ is given by

$$
\dot{\mathcal{J}}_{\chi_{\varepsilon}}\left(u_{\varepsilon}, \theta_{\varepsilon}\right)=\int_{\Omega} \Sigma_{\varepsilon} \cdot \nabla V-\int_{\Omega} Q_{\varepsilon}\left(\dot{\theta}_{\varepsilon}\right) \cdot \nabla\left(u_{\varepsilon}-u\right)^{s},
$$

where $V$ is the shape change velocity field defined in $\Omega$ that satisfies $\left.V\right|_{\partial \Omega}=0$ and $\left.V\right|_{\partial B_{\varepsilon}}=$ $-n ; \dot{\theta}_{\varepsilon}$ is the material derivative of the temperature field and $\Sigma_{\varepsilon}$ is a generalization of the classical Eshelby momentum-energy tensor [8], given - for this particular case - by

$\Sigma_{\varepsilon}:=\frac{1}{2}\left(\left(S_{\varepsilon}\left(u_{\varepsilon}, \theta_{\varepsilon}\right)-Q_{\varepsilon}\left(\theta_{\varepsilon}\right)\right) \cdot \nabla u_{\varepsilon}^{s}\right) I-\left(\nabla u_{\varepsilon}\right)^{\top} S_{\varepsilon}\left(u_{\varepsilon}, \theta_{\varepsilon}\right)+\left[\left(q_{\varepsilon}\left(\theta_{\varepsilon}\right) \cdot \nabla \varphi_{\varepsilon}\right) I-2 q_{\varepsilon}\left(\theta_{\varepsilon}\right) \otimes_{s} \nabla \varphi_{\varepsilon}\right]$,

with $u_{\varepsilon}, \theta_{\varepsilon}$ and $\varphi_{\varepsilon}$ denoting the solutions to (23), (26) and to the auxiliary problem (30).

Proof. By making use of Reynolds' Transport Theorem [11, 20] we obtain the identities

$$
\begin{aligned}
\left(\int_{\Omega} \sigma_{\varepsilon}\left(u_{\varepsilon}\right) \cdot \nabla u_{\varepsilon}^{s}\right)^{\prime}= & \int_{\Omega}\left(2 \sigma_{\varepsilon}\left(u_{\varepsilon}\right) \cdot \nabla \dot{u}_{\varepsilon}^{s}-2 \sigma_{\varepsilon}\left(u_{\varepsilon}\right) \cdot\left(\nabla u_{\varepsilon} \nabla V\right)^{s}+\left(\sigma_{\varepsilon}\left(u_{\varepsilon}\right) \cdot \nabla u_{\varepsilon}^{s}\right) \operatorname{div}\right. \text { (3) .) } \\
\left(\int_{\Omega} Q_{\varepsilon}\left(\theta_{\varepsilon}\right) \cdot \nabla u_{\varepsilon}^{s}\right)^{\prime}= & \int_{\Omega}\left(Q_{\varepsilon}\left(\theta_{\varepsilon}\right) \cdot \nabla \dot{u}_{\varepsilon}^{s}-Q_{\varepsilon}\left(\theta_{\varepsilon}\right) \cdot\left(\nabla u_{\varepsilon} \nabla V\right)^{s}+\left(Q_{\varepsilon}\left(\theta_{\varepsilon}\right) \cdot \nabla u_{\varepsilon}^{s}\right) \operatorname{div} V\right. \\
& +Q_{\varepsilon}\left(\dot{\theta}_{\varepsilon}\right) \cdot \nabla u_{\varepsilon}^{s} .
\end{aligned}
$$

Then, by considering the above results in (31), the shape derivative of the functional $\mathcal{J}_{\chi_{\varepsilon}}\left(u_{\varepsilon}, \theta_{\varepsilon}\right)$ is given by

$$
\begin{aligned}
\dot{\mathcal{J}}_{\bar{\varepsilon}}\left(u_{\varepsilon}, \theta_{\varepsilon}\right) & =\int_{\Omega}\left(\frac{1}{2}\left(\left(S_{\varepsilon}\left(u_{\varepsilon}, \theta_{\varepsilon}\right)-Q_{\varepsilon}\left(\theta_{\varepsilon}\right)\right) \cdot \nabla u_{\varepsilon}^{s}\right) I-\left(\nabla u_{\varepsilon}\right)^{\top} S_{\varepsilon}\left(u_{\varepsilon}, \theta_{\varepsilon}\right)\right) \cdot \nabla V \\
& -\int_{\Omega} Q_{\varepsilon}\left(\dot{\theta}_{\varepsilon}\right) \cdot \nabla u_{\varepsilon}^{s}+\int_{\Omega} S_{\varepsilon}\left(u_{\varepsilon}, \theta_{\varepsilon}\right) \cdot \nabla \dot{u}_{\varepsilon}^{s}-\int_{\Gamma_{N_{M}}} \bar{t} \cdot \dot{u}_{\varepsilon}
\end{aligned}
$$

Since $\dot{u}_{\varepsilon} \in \mathcal{U}_{\varepsilon}^{M}$, see [20], the terms in $\dot{u}_{\varepsilon}$ satisfy the state equation (23), then

$$
\begin{aligned}
\dot{\mathcal{J}}_{\chi_{\varepsilon}}\left(u_{\varepsilon}, \theta_{\varepsilon}\right) & =\int_{\Omega}\left(\frac{1}{2}\left(\left(S_{\varepsilon}\left(u_{\varepsilon}, \theta_{\varepsilon}\right)-Q_{\varepsilon}\left(\theta_{\varepsilon}\right)\right) \cdot \nabla u_{\varepsilon}^{s}\right) I-\left(\nabla u_{\varepsilon}\right)^{\top} S_{\varepsilon}\left(u_{\varepsilon}, \theta_{\varepsilon}\right)\right) \cdot \nabla V \\
& -\int_{\Omega} Q_{\varepsilon}\left(\dot{\theta}_{\varepsilon}\right) \cdot \nabla u_{\varepsilon}^{s}
\end{aligned}
$$

Now, adding the term $\pm \int_{\Omega} Q_{\varepsilon}\left(\dot{\theta}_{\varepsilon}\right) \cdot \nabla u^{s}$ in the above result, the derivative $\dot{\mathcal{J}}_{\chi_{\varepsilon}}\left(u_{\varepsilon}, \theta_{\varepsilon}\right)$ can be written alternatively as

$$
\begin{aligned}
\dot{\mathcal{J}}_{\chi_{\varepsilon}}\left(u_{\varepsilon}, \theta_{\varepsilon}\right) & =\int_{\Omega}\left(\frac{1}{2}\left(\left(S_{\varepsilon}\left(u_{\varepsilon}, \theta_{\varepsilon}\right)-Q_{\varepsilon}\left(\theta_{\varepsilon}\right)\right) \cdot \nabla u_{\varepsilon}^{s}\right) I-\left(\nabla u_{\varepsilon}\right)^{\top} S_{\varepsilon}\left(u_{\varepsilon}, \theta_{\varepsilon}\right)\right) \cdot \nabla V \\
& -\int_{\Omega} Q_{\varepsilon}\left(\dot{\theta}_{\varepsilon}\right) \cdot \nabla\left(u_{\varepsilon}-u\right)^{s}-\int_{\Omega} Q_{\varepsilon}\left(\dot{\theta}_{\varepsilon}\right) \cdot \nabla u^{s}
\end{aligned}
$$

On the other hand, the deriative of the state equation (26) with respect to the parameter $\varepsilon$ is given by

$$
\int_{\Omega} q_{\varepsilon}\left(\dot{\theta}_{\varepsilon}\right) \cdot \nabla \eta=-\int_{\Omega}\left[\left(q_{\varepsilon}\left(\theta_{\varepsilon}\right) \cdot \nabla \eta\right) I-2 q_{\varepsilon}\left(\theta_{\varepsilon}\right) \otimes_{s} \nabla \eta\right] \cdot \nabla V \quad \forall \eta \in \mathcal{V}_{\varepsilon}^{T}
$$


Next, taking $\eta=\varphi_{\varepsilon}$ in the above expression, we obtain

$$
\int_{\Omega} q_{\varepsilon}\left(\dot{\theta}_{\varepsilon}\right) \cdot \nabla \varphi_{\varepsilon}=-\int_{\Omega}\left[\left(q_{\varepsilon}\left(\theta_{\varepsilon}\right) \cdot \nabla \varphi_{\varepsilon}\right) I-2 q_{\varepsilon}\left(\theta_{\varepsilon}\right) \otimes_{s} \nabla \varphi_{\varepsilon}\right] \cdot \nabla V,
$$

and tacking $\eta=\dot{\theta}_{\varepsilon}$ in the auxiliary problem (30), we obtain

$$
\int_{\Omega} q_{\varepsilon}\left(\varphi_{\varepsilon}\right) \cdot \nabla \dot{\theta}_{\varepsilon}=\int_{\Omega} Q_{\varepsilon}\left(\dot{\theta}_{\varepsilon}\right) \cdot \nabla u^{s} .
$$

By using the definition of the heat flux operator (27) and comparing the two last expressions, the following identity holds

$$
\int_{\Omega} Q_{\varepsilon}\left(\dot{\theta}_{\varepsilon}\right) \cdot \nabla u^{s}=-\int_{\Omega}\left[\left(q_{\varepsilon}\left(\theta_{\varepsilon}\right) \cdot \nabla \varphi_{\varepsilon}\right) I-2 q_{\varepsilon}\left(\theta_{\varepsilon}\right) \otimes_{s} \nabla \varphi_{\varepsilon}\right] \cdot \nabla V
$$

From the above result, the derivative of the shape functional $\mathcal{J}_{\chi_{\varepsilon}}\left(u_{\varepsilon}, \theta_{\varepsilon}\right)$ can be written equivalently in the following form:

$$
\begin{aligned}
\dot{\mathcal{J}}_{\chi_{\varepsilon}}\left(u_{\varepsilon}, \theta_{\varepsilon}\right) & =\int_{\Omega}\left(\frac{1}{2}\left(\left(S_{\varepsilon}\left(u_{\varepsilon}, \theta_{\varepsilon}\right)-Q_{\varepsilon}\left(\theta_{\varepsilon}\right)\right) \cdot \nabla u_{\varepsilon}^{s}\right) I-\left(\nabla u_{\varepsilon}\right)^{\top} S_{\varepsilon}\left(u_{\varepsilon}, \theta_{\varepsilon}\right)\right) \cdot \nabla V \\
& +\int_{\Omega}\left[\left(q_{\varepsilon}\left(\theta_{\varepsilon}\right) \cdot \nabla \varphi_{\varepsilon}\right) I-2 q_{\varepsilon}\left(\theta_{\varepsilon}\right) \otimes_{s} \nabla \varphi_{\varepsilon}\right] \cdot \nabla V \\
& -\int_{\Omega} Q_{\varepsilon}\left(\dot{\theta}_{\varepsilon}\right) \cdot \nabla\left(u_{\varepsilon}-u\right)^{s}
\end{aligned}
$$

which leads to the result with $\Sigma_{\varepsilon}$ given by (33).

Proposition 2 Let $\mathcal{J}_{\chi_{\varepsilon}}\left(u_{\varepsilon}, \theta_{\varepsilon}\right)$ be the functional defined by (20). Then, its derivative with respect to the small parameter $\varepsilon$ is given by

$$
\dot{\mathcal{J}}_{\chi_{\varepsilon}}\left(u_{\varepsilon}, \theta_{\varepsilon}\right)=-\int_{\partial B_{\varepsilon}} \llbracket \Sigma_{\varepsilon} \rrbracket n \cdot n-\int_{\Omega} Q_{\varepsilon}\left(\theta_{\varepsilon}^{\prime}\right) \cdot \nabla\left(u_{\varepsilon}-u\right)^{s},
$$

where $V$ is the shape change velocity field defined in $\Omega$ that satisfies $\left.V\right|_{\partial \Omega}=0$ and $\left.V\right|_{\partial B_{\varepsilon}}=$ $-n ; \theta_{\varepsilon}^{\prime}$ is the spatial derivative of the temperature field and $\Sigma_{\varepsilon}$ is a generalization of the classical Eshelby momentum-energy tensor presented in (33).

Proof. By making use of the Reynolds' Transport Theorem [11, 20], we obtain the following identities:

$$
\begin{aligned}
\left(\int_{\Omega} \sigma_{\varepsilon}\left(u_{\varepsilon}\right) \cdot \nabla u_{\varepsilon}^{s}\right)^{\cdot} & =\int_{\Omega} 2\left(\sigma_{\varepsilon}\left(u_{\varepsilon}\right) \cdot \nabla \dot{u}_{\varepsilon}^{s}+\operatorname{div}\left(\sigma_{\varepsilon}\left(u_{\varepsilon}\right)\right) \cdot\left(\nabla u_{\varepsilon}\right) V\right) \\
& +\int_{\partial \Omega}\left[\left(\sigma_{\varepsilon}\left(u_{\varepsilon}\right) \cdot \nabla u_{\varepsilon}^{s}\right) I-2\left(\nabla u_{\varepsilon}\right)^{\top} \sigma_{\varepsilon}\left(u_{\varepsilon}\right)\right] n \cdot V \\
& +\int_{\partial B_{\varepsilon}} \llbracket\left(\sigma_{\varepsilon}\left(u_{\varepsilon}\right) \cdot \nabla u_{\varepsilon}^{s}\right) I-2\left(\nabla u_{\varepsilon}\right)^{\top} \sigma_{\varepsilon}\left(u_{\varepsilon}\right) \rrbracket n \cdot V \\
\left(\int_{\Omega} Q_{\varepsilon}\left(\theta_{\varepsilon}\right) \cdot \nabla u_{\varepsilon}^{s}\right) \cdot & =\int_{\Omega}\left(Q_{\varepsilon}\left(\theta_{\varepsilon}\right) \cdot \nabla \dot{u}_{\varepsilon}^{s}+Q_{\varepsilon}\left(\theta_{\varepsilon}^{\prime}\right) \cdot \nabla u_{\varepsilon}^{s}\right)+\int_{\Omega} \operatorname{div}\left(Q_{\varepsilon}\left(\theta_{\varepsilon}\right)\right) \cdot\left(\nabla u_{\varepsilon}\right) V \\
& -\int_{\partial \Omega}\left[\left(\nabla u_{\varepsilon}\right)^{\top} Q_{\varepsilon}\left(\theta_{\varepsilon}\right)-\left(Q_{\varepsilon}\left(\theta_{\varepsilon}\right) \cdot \nabla u_{\varepsilon}^{s}\right) I\right] n \cdot V \\
& -\int_{\partial B_{\varepsilon}} \llbracket\left(\nabla u_{\varepsilon}\right)^{\top} Q_{\varepsilon}\left(\theta_{\varepsilon}\right)-\left(Q_{\varepsilon}\left(\theta_{\varepsilon}\right) \cdot \nabla u_{\varepsilon}^{s}\right) I \rrbracket n \cdot V
\end{aligned}
$$


Introducing the above expressions in the definitions of the shape derivative (31) and taking into account that: (i) $\dot{u}_{\varepsilon} \in \mathcal{U}_{\varepsilon}^{M}$, see [20], the terms in $\dot{u}_{\varepsilon}$ satisfy the state equation (23); (ii) $\operatorname{div} S_{\varepsilon}\left(u_{\varepsilon}, \theta_{\varepsilon}\right)=0$ in $\Omega$; (iii) adding the term $\pm \int_{\Omega} Q_{\varepsilon}\left(\theta_{\varepsilon}^{\prime}\right) \cdot \nabla u^{s}$; and (iv) the shape change velocity field $V$ defined in $\Omega$ satisfies $\left.V\right|_{\partial \Omega}=0$ and $\left.V\right|_{\partial B_{\varepsilon}}=-n$; then

$$
\begin{aligned}
\dot{\mathcal{J}}_{\chi_{\varepsilon}}\left(u_{\varepsilon}, \theta_{\varepsilon}\right)= & -\int_{\partial B_{\varepsilon}} \llbracket \frac{1}{2}\left(\left(S_{\varepsilon}\left(u_{\varepsilon}, \theta_{\varepsilon}\right)-Q_{\varepsilon}\left(\theta_{\varepsilon}\right)\right) \cdot \nabla u_{\varepsilon}^{s}\right) I-\left(\nabla u_{\varepsilon}\right)^{\top} S_{\varepsilon}\left(u_{\varepsilon}, \theta_{\varepsilon}\right) \rrbracket n \cdot n \\
& -\int_{\Omega} Q_{\varepsilon}\left(\theta_{\varepsilon}^{\prime}\right) \cdot \nabla\left(u_{\varepsilon}-u\right)^{s}-\int_{\Omega} Q_{\varepsilon}\left(\theta_{\varepsilon}^{\prime}\right) \cdot \nabla u^{s} .
\end{aligned}
$$

By using the relation between the material and spatial derivatives of the temperature field, the above expression can be written as,

$$
\begin{aligned}
\dot{\mathcal{J}}_{\chi_{\varepsilon}}\left(u_{\varepsilon}, \theta_{\varepsilon}\right)= & -\int_{\partial B_{\varepsilon}} \llbracket \frac{1}{2}\left(\left(S_{\varepsilon}\left(u_{\varepsilon}, \theta_{\varepsilon}\right)-Q_{\varepsilon}\left(\theta_{\varepsilon}\right)\right) \cdot \nabla u_{\varepsilon}^{s}\right) I-\left(\nabla u_{\varepsilon}\right)^{\top} S_{\varepsilon}\left(u_{\varepsilon}, \theta_{\varepsilon}\right) \rrbracket n \cdot n \\
& -\int_{\Omega} Q_{\varepsilon}\left(\theta_{\varepsilon}^{\prime}\right) \cdot \nabla\left(u_{\varepsilon}-u\right)^{s}-\int_{\Omega} Q_{\varepsilon}\left(\dot{\theta}_{\varepsilon}\right) \cdot \nabla u^{s}+\int_{\Omega} Q_{\varepsilon}\left(\nabla \theta_{\varepsilon} \cdot n\right) \cdot \nabla u^{s}(48)
\end{aligned}
$$

On the other hand, the derivative of the state equation (26) with respect to parameter $\varepsilon$ is given by

$$
\int_{\Omega} q_{\varepsilon}\left(\dot{\theta}_{\varepsilon}\right) \cdot \nabla \eta=-\int_{\Omega}\left[\left(q_{\varepsilon}\left(\theta_{\varepsilon}\right) \cdot \nabla \eta\right) I-2 q_{\varepsilon}\left(\theta_{\varepsilon}\right) \otimes_{s} \nabla \eta\right] \cdot \nabla V \quad \forall \eta \in \mathcal{V}_{\varepsilon}^{T}
$$

Next, tacking $\eta=\varphi_{\varepsilon}$ in the above expression, we obtain

$$
\int_{\Omega} q_{\varepsilon}\left(\dot{\theta}_{\varepsilon}\right) \cdot \nabla \varphi_{\varepsilon}=-\int_{\Omega}\left[\left(q_{\varepsilon}\left(\theta_{\varepsilon}\right) \cdot \nabla \varphi_{\varepsilon}\right) I-2 q_{\varepsilon}\left(\theta_{\varepsilon}\right) \otimes_{s} \nabla \varphi_{\varepsilon}\right] \cdot \nabla V
$$

and tacking $\eta=\dot{\theta}_{\varepsilon}$ in the auxiliary problem (30), we obtain

$$
\int_{\Omega} q_{\varepsilon}\left(\varphi_{\varepsilon}\right) \cdot \nabla \dot{\theta}_{\varepsilon}=\int_{\Omega} Q_{\varepsilon}\left(\dot{\theta}_{\varepsilon}\right) \cdot \nabla u^{s}
$$

By using the definition of the heat flux operator (27) and comparing the two last expressions, the following identity holds

$$
\int_{\Omega} Q_{\varepsilon}\left(\dot{\theta}_{\varepsilon}\right) \cdot \nabla u^{s}=-\int_{\Omega}\left[\left(q_{\varepsilon}\left(\theta_{\varepsilon}\right) \cdot \nabla \varphi_{\varepsilon}\right) I-2 q_{\varepsilon}\left(\theta_{\varepsilon}\right) \otimes_{s} \nabla \varphi_{\varepsilon}\right] \cdot \nabla V .
$$

From the above result, the derivative of the shape functional $\mathcal{J}_{\chi_{\varepsilon}}\left(u_{\varepsilon}, \theta_{\varepsilon}\right)$ can be written equivalently in the following form,

$$
\begin{aligned}
\dot{\mathcal{J}}_{\chi_{\varepsilon}}\left(u_{\varepsilon}, \theta_{\varepsilon}\right)= & -\int_{\partial B_{\varepsilon}} \llbracket \frac{1}{2}\left(\left(S_{\varepsilon}\left(u_{\varepsilon}, \theta_{\varepsilon}\right)-Q_{\varepsilon}\left(\theta_{\varepsilon}\right)\right) \cdot \nabla u_{\varepsilon}^{s}\right) I-\left(\nabla u_{\varepsilon}\right)^{\top} S_{\varepsilon}\left(u_{\varepsilon}, \theta_{\varepsilon}\right) \rrbracket n \cdot n \\
& +\int_{\Omega}\left[\left(q_{\varepsilon}\left(\theta_{\varepsilon}\right) \cdot \nabla \varphi_{\varepsilon}\right) I-2 q_{\varepsilon}\left(\theta_{\varepsilon}\right) \otimes_{s} \nabla \varphi_{\varepsilon}\right] \cdot \nabla V \\
& -\int_{\Omega} Q_{\varepsilon}\left(\theta_{\varepsilon}^{\prime}\right) \cdot \nabla\left(u_{\varepsilon}-u\right)^{s}+\int_{\Omega} Q_{\varepsilon}\left(\nabla \theta_{\varepsilon} \cdot n\right) \cdot \nabla u^{s} .
\end{aligned}
$$


By integrating by parts the second term in the above expression and using the definition of the Eshelby's tensor $\Sigma_{\varepsilon}$, we have

$$
\begin{aligned}
\dot{\mathcal{J}}_{\chi_{\varepsilon}}\left(u_{\varepsilon}, \theta_{\varepsilon}\right)= & -\int_{\partial B_{\varepsilon}} \llbracket \Sigma_{\varepsilon} \rrbracket n \cdot n-\int_{\Omega} Q_{\varepsilon}\left(\theta_{\varepsilon}^{\prime}\right) \cdot \nabla\left(u_{\varepsilon}-u\right)^{s} \\
& -\int_{\Omega} \operatorname{div}\left[\left(q_{\varepsilon}\left(\theta_{\varepsilon}\right) \cdot \nabla \varphi_{\varepsilon}\right) I-2 q_{\varepsilon}\left(\theta_{\varepsilon}\right) \otimes_{s} \nabla \varphi_{\varepsilon}\right] \cdot V \\
& +\int_{\Omega} Q_{\varepsilon}\left(\nabla \theta_{\varepsilon} \cdot V\right) \cdot \nabla u^{s} .
\end{aligned}
$$

Taking into account the state equation (26) and the auxiliary problem (30), we observe that the second term in the above expression satisfies the following identity

$$
\int_{\Omega} \operatorname{div}\left[\left(q_{\varepsilon}\left(\theta_{\varepsilon}\right) \cdot \nabla \varphi_{\varepsilon}\right) I-2 q_{\varepsilon}\left(\theta_{\varepsilon}\right) \otimes_{s} \nabla \varphi_{\varepsilon}\right] \cdot V=\int_{\Omega} Q_{\varepsilon}\left(\nabla \theta_{\varepsilon} \cdot V\right) \cdot \nabla u^{s}
$$

Then, the lats two terms in (54) vanish, leading to the result.

Corollary 3 By considering the relation between the material and spatial derivative of the temperature field, (32) can be written as:

$$
\dot{\mathcal{J}}_{\chi_{\varepsilon}}\left(u_{\varepsilon}, \theta_{\varepsilon}\right)=\int_{\Omega} \Sigma_{\varepsilon} \cdot \nabla V-\int_{\Omega} Q_{\varepsilon}\left(\theta_{\varepsilon}^{\prime}\right) \cdot \nabla\left(u_{\varepsilon}-u\right)^{s}-\int_{\Omega} Q_{\varepsilon}\left(\nabla \theta_{\varepsilon} \cdot V\right) \cdot \nabla\left(u_{\varepsilon}-u\right)^{s} .
$$

By integrating by part the firt term of the above expression and using the restriccion of the velocity field $V$ on the boundaries $\partial \Omega$ and $\partial B_{\varepsilon}$, we obtain

$\dot{\mathcal{J}}_{\chi_{\varepsilon}}\left(u_{\varepsilon}, \theta_{\varepsilon}\right)=-\int_{\partial B_{\varepsilon}} \llbracket \Sigma_{\varepsilon} \rrbracket n \cdot n-\int_{\Omega} \operatorname{div} \Sigma_{\varepsilon} \cdot V-\int_{\Omega} Q_{\varepsilon}\left(\theta_{\varepsilon}^{\prime}\right) \cdot \nabla\left(u_{\varepsilon}-u\right)^{s}-\int_{\Omega} Q_{\varepsilon}\left(\nabla \theta_{\varepsilon} \cdot V\right) \cdot \nabla\left(u_{\varepsilon}-u\right)^{s}$.

By comparing (44) with (57) and recalling that both identities are valid for all $V \in \Omega$, the follow result holds true

$$
\int_{\Omega}\left(\operatorname{div}\left(\Sigma_{\varepsilon}\right)+\gamma_{\varepsilon}^{M} \gamma_{\varepsilon}^{C}\left(\mathbb{C} B \cdot \nabla\left(u_{\varepsilon}-u\right)^{s}\right) \nabla \theta_{\varepsilon}\right) \cdot V=0 \quad \forall V \in \Omega,
$$

Thus, the equation for the balance of the configurational forces [12] can be written as:

$$
\operatorname{div}\left(\Sigma_{\varepsilon}\right)=-\gamma_{\varepsilon}^{M} \gamma_{\varepsilon}^{C}\left(\mathbb{C} B \cdot \nabla\left(u_{\varepsilon}-u\right)^{s}\right) \nabla \theta_{\varepsilon} \quad \text { in } \quad \Omega .
$$

To analytically solve the integrals expression of the derivative $\dot{\mathcal{J}}_{\chi_{\varepsilon}}\left(u_{\varepsilon}, \theta_{\varepsilon}\right)$ it is necessary to perform an asymptotic analysis of the solutions of the PDE's involved in these coupled problems. In order to simplify the analysys, let us use the linearity property of the shape functional with respect to the solution of the thermal problem (26) and split the analysis in two cases: (i) $\gamma^{T}=1$ and (ii) $\gamma^{M}=\gamma^{C}=1$.

\subsection{Case $\gamma^{T}=1$}

For this particular case, $\gamma^{T}=1$, we have that the temperature field is not perturbed by the presence of the inclusion $B_{\varepsilon}$ in the mechanical problem. Then, the temperature for the 
unperturbed and perturbed problems coincides, i.e. $\theta_{\varepsilon}=\theta$. Thus, the derivative of the shape functional can be written as:

$$
\dot{\mathcal{J}}_{\chi_{\varepsilon}}\left(u_{\varepsilon}, \theta\right)=-\int_{\partial B_{\varepsilon}} \llbracket \Sigma_{\varepsilon} \rrbracket n \cdot n=-\int_{\partial B_{\varepsilon}} \llbracket \frac{1}{2}\left(\left(S_{\varepsilon}\left(u_{\varepsilon}, \theta\right)-Q_{\varepsilon}(\theta)\right) \cdot \nabla u_{\varepsilon}^{s}\right) I-\left(\nabla u_{\varepsilon}\right)^{\top} S_{\varepsilon}\left(u_{\varepsilon}, \theta\right) \rrbracket n \cdot n .
$$

Considering a curvilinear coordinate system $(t, n)$ defined on the boundary of the inclusion $\partial B_{\varepsilon}$, the jump condition of the stress field $S_{\varepsilon}\left(u_{\varepsilon}, \theta\right)$ in the boundary $\partial B_{\varepsilon}$ can be written, tacking into account the orthogonality of the normal $(n)$ and tangential $(t)$ vectors, as:

$$
\llbracket S_{\varepsilon}\left(u_{\varepsilon}, \theta\right) \rrbracket n=\llbracket S_{\varepsilon}^{n n}\left(u_{\varepsilon}, \theta\right) \rrbracket n+\llbracket S_{\varepsilon}^{t n}\left(u_{\varepsilon}, \theta\right) \rrbracket t=0,
$$

which leads to the following result,

$$
\llbracket S_{\varepsilon}^{n n}\left(u_{\varepsilon}, \theta\right) \rrbracket=0 \quad \text { and } \quad \llbracket S_{\varepsilon}^{t n}\left(u_{\varepsilon}, \theta\right) \rrbracket=0 \quad \text { on } \partial B_{\varepsilon} .
$$

In the same way, the continuity condition of the displacement field defined on the boundary $\partial B_{\varepsilon}$ results in the following relations

$$
\llbracket u_{\varepsilon} \rrbracket=0 \quad \Rightarrow \quad \llbracket \partial_{t} u_{\varepsilon} \rrbracket=0 \quad \text { on } \partial B_{\varepsilon} .
$$

The above continuity relations implies the continuity of the tangential component of the deformation tensor $\nabla u_{\varepsilon}^{s}$,

$$
\llbracket u_{\varepsilon} \rrbracket=0 \quad \Rightarrow \quad \llbracket\left(\nabla u_{\varepsilon}^{s}\right)^{t t} \rrbracket=0 \quad \text { on } \partial B_{\varepsilon} .
$$

In view of the above decomposition, it is possible to analyze each term of (60) separately:

$$
\begin{aligned}
& \llbracket\left(S_{\varepsilon}\left(u_{\varepsilon}, \theta\right) \cdot \nabla u_{\varepsilon}^{s}\right) I \rrbracket n \cdot n=\llbracket S_{\varepsilon}\left(u_{\varepsilon}, \theta\right) \cdot \nabla u_{\varepsilon}^{s} \rrbracket
\end{aligned}
$$

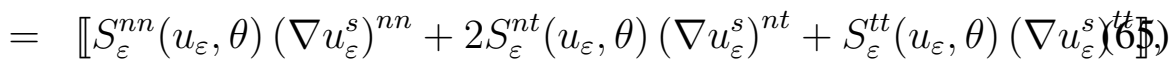

$$
\begin{aligned}
& \llbracket\left(\nabla u_{\varepsilon}\right)^{\top} S_{\varepsilon}\left(u_{\varepsilon}, \theta\right) \rrbracket n \cdot n=\llbracket S_{\varepsilon}^{n n}\left(u_{\varepsilon}, \theta\right) \partial_{n} u_{\varepsilon}^{n}+S_{\varepsilon}^{t n}\left(u_{\varepsilon}, \theta\right) \partial_{n} u_{\varepsilon}^{t} \rrbracket, \\
& \llbracket\left(Q_{\varepsilon}(\theta) \cdot \nabla u_{\varepsilon}^{s}\right) I \rrbracket n \cdot n=\llbracket Q_{\varepsilon}(\theta) \cdot \nabla u_{\varepsilon}^{s} \rrbracket \\
& =\llbracket Q_{\varepsilon}^{n n}(\theta)\left(\nabla u_{\varepsilon}^{s}\right)^{n n}+2 Q_{\varepsilon}^{n t}(\theta)\left(\nabla u_{\varepsilon}^{s}\right)^{n t}+Q_{\varepsilon}^{t t}(\theta)\left(\nabla u_{\varepsilon}^{s}\right)^{t t} \rrbracket,
\end{aligned}
$$

where $\partial_{n} u_{\varepsilon}^{t}=\left(\nabla u_{\varepsilon}\right)^{t n}$. With the above continuity properties, the expression (65) can be written as:

$$
\llbracket S_{\varepsilon}\left(u_{\varepsilon}, \theta\right) \cdot \nabla u_{\varepsilon}^{s} \rrbracket=S_{\varepsilon}^{n n}\left(u_{\varepsilon}, \theta\right) \llbracket\left(\nabla u_{\varepsilon}^{s}\right)^{n n} \rrbracket+S^{t n}\left(u_{\varepsilon}, \theta\right) \llbracket \partial_{n} u_{\varepsilon}^{t} \rrbracket+\left(\nabla u_{\varepsilon}^{s}\right)^{t t} \llbracket S_{\varepsilon}^{t t}\left(u_{\varepsilon}, \theta\right) \rrbracket .
$$

By using the same continuity properties, (66) can be re-written as:

$$
\llbracket\left(\nabla u_{\varepsilon}\right)^{\top} S_{\varepsilon}\left(u_{\varepsilon}, \theta\right) \rrbracket n \cdot n=S_{\varepsilon}^{n n}\left(u_{\varepsilon}, \theta\right) \llbracket\left(\nabla u_{\varepsilon}^{s}\right)^{n n} \rrbracket+S_{\varepsilon}^{t n}\left(u_{\varepsilon}, \theta\right) \llbracket \partial_{n} u_{\varepsilon}^{t} \rrbracket .
$$

By considering an isotropic thermal expansion, i.e. $Q^{n n}(\theta)=Q^{t t}(\theta)$ and $Q^{n t}(\theta)=0$, from (67) we have that

$$
\llbracket Q_{\varepsilon}(\theta) \cdot \nabla u_{\varepsilon}^{s} \rrbracket=Q^{n n}(\theta)\left(\llbracket \gamma_{\varepsilon}^{C} \gamma_{\varepsilon}^{C}\left(\nabla u_{\varepsilon}^{s}\right)^{n n} \rrbracket+\left(1-\gamma^{M} \gamma^{C}\right)\left(\nabla u_{\varepsilon}^{s}\right)^{t t}\right) .
$$


Then, the operator of the normal jump of the Eshely tensor on the boundary of the perturbation $B_{\varepsilon}$ can be written as:

$$
\begin{aligned}
2 \llbracket \Sigma_{\varepsilon} \rrbracket n \cdot n & =\left(\nabla u_{\varepsilon}^{s}\right)^{t t} \llbracket S_{\varepsilon}^{t t}\left(u_{\varepsilon}, \theta\right) \rrbracket-S_{\varepsilon}^{n n}\left(u_{\varepsilon}, \theta\right) \llbracket\left(\nabla u_{\varepsilon}^{s}\right)^{n n} \rrbracket-S_{\varepsilon}^{n t}\left(u_{\varepsilon}, \theta\right) \llbracket \partial_{n} u_{\varepsilon}^{t} \rrbracket \\
& -Q^{n n}(\theta)\left(\llbracket \gamma_{\varepsilon}^{M} \gamma_{\varepsilon}^{C}\left(\nabla u_{\varepsilon}^{s}\right)^{n n} \rrbracket+\left(1-\gamma^{M} \gamma^{C}\right)\left(\nabla u_{\varepsilon}^{s}\right)^{t t}\right) .
\end{aligned}
$$

Note that, the first part of the normal jump of the Eshelby tensor is classical presented in terms of the mechanical deformation $\nabla u_{\varepsilon}^{s}$. The second part of this jump represents the explicit contribution of thermal problem through the thermal expansion parameter. Finally, the derivative of the shape functional $\mathcal{J}_{\chi_{\varepsilon}}\left(u_{\varepsilon}, \theta\right)$ can be written as:

$$
\begin{aligned}
\dot{\mathcal{J}}_{\chi_{\varepsilon}}\left(u_{\varepsilon}, \theta\right)= & -\frac{1}{2} \int_{\partial B_{\varepsilon}}\left(\nabla u_{\varepsilon}^{s}\right)^{t t} \llbracket S_{\varepsilon}^{t t}\left(u_{\varepsilon}, \theta\right) \rrbracket-S_{\varepsilon}^{n n}\left(u_{\varepsilon}, \theta\right) \llbracket\left(\nabla u_{\varepsilon}^{s}\right)^{n n} \rrbracket-S_{\varepsilon}^{n t}\left(u_{\varepsilon}, \theta\right) \llbracket \partial_{n} u_{\varepsilon}^{t} \rrbracket \\
& -Q^{n n}(\theta)\left(\llbracket \gamma_{\varepsilon}^{M} \gamma_{\varepsilon}^{C}\left(\nabla u_{\varepsilon}^{s}\right)^{n n} \rrbracket+\left(1-\gamma^{M} \gamma^{C}\right)\left(\nabla u_{\varepsilon}^{s}\right)^{t t}\right) .
\end{aligned}
$$

In order to obtain an explicit expression for the perturbed stress field, we consider the following ansatz for the displacement field $u_{\varepsilon}$ :

$$
u_{\varepsilon}(x)=u(x)+w_{\varepsilon}(x / \varepsilon)+\widetilde{u}_{\varepsilon}(x),
$$

where $w_{\varepsilon}$ is such that it decays to zero at the infinity, i.e., $w_{\varepsilon} \rightarrow 0$ at $\infty$. Then, the mechanical stress satisfies the identity

$$
\sigma_{\varepsilon}\left(u_{\varepsilon}\right)=\gamma_{\varepsilon}^{M} \mathbb{C} \nabla u^{s}+\gamma_{\varepsilon}^{M} \mathbb{C} \nabla w_{\varepsilon}^{s}+\gamma_{\varepsilon}^{M} \mathbb{C} \nabla \widetilde{u}_{\varepsilon}^{s} .
$$

Moreover, by introducing the term $-Q_{\varepsilon}(\theta)$ at both sides of the above expression, the stress field associated to the perturbed domain $S_{\varepsilon}\left(u_{\varepsilon}, \theta\right)$ admits the following asymptotic expansion

$$
S_{\varepsilon}\left(u_{\varepsilon}, \theta\right)=\gamma_{\varepsilon}^{M} \sigma(u)+\sigma_{\varepsilon}\left(w_{\varepsilon}\right)+\sigma_{\varepsilon}\left(\widetilde{u}_{\varepsilon}\right)-\gamma_{\varepsilon}^{M} \gamma_{\varepsilon}^{C} Q(\theta),
$$

where $\sigma_{\varepsilon}\left(w_{\varepsilon}\right)$ is the solution of the exterior problem

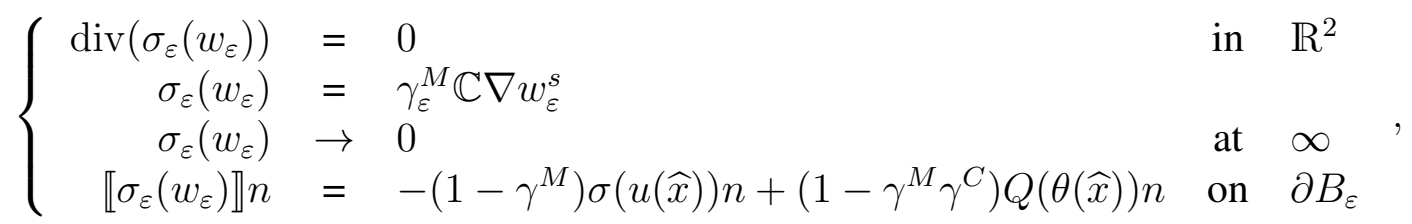

and the residue $\widetilde{u}_{\varepsilon}$ satisfies the equation

$$
\left\{\begin{array}{rlrl}
\operatorname{div}\left(\sigma_{\varepsilon}\left(\widetilde{u}_{\varepsilon}\right)\right) & =0 & & \text { in } \quad \backslash \backslash \overline{B_{\varepsilon}} \\
\operatorname{div}\left(\sigma_{\varepsilon}\left(\widetilde{u}_{\varepsilon}\right)\right) & =\left(1-\gamma^{C}\right) \gamma^{M} \mathbb{C} B \nabla \theta & & \text { in } B_{\varepsilon} \\
\sigma_{\varepsilon}\left(\widetilde{u}_{\varepsilon}\right) & =\gamma_{\varepsilon}^{M} \mathbb{C} \nabla \widetilde{u}_{\varepsilon}^{s} & & \\
\widetilde{u}_{\varepsilon} & =-w_{\varepsilon} & & \text { on } \partial \Gamma_{D} \\
\sigma_{\varepsilon}\left(\widetilde{u}_{\varepsilon}\right) & =-\sigma_{\varepsilon}\left(w_{\varepsilon}\right) & \text { on } \partial \Gamma_{N} \\
\llbracket \sigma_{\varepsilon}\left(\widetilde{u}_{\varepsilon}\right) \rrbracket n & =\varepsilon\left(1-\gamma^{M}\right)(\nabla \sigma(u(\zeta)) n) n-\varepsilon\left(1-\gamma^{M} \gamma^{C}\right)(\nabla Q(\theta(\xi)) n) n & & \text { on } \partial B_{\varepsilon}
\end{array},\right.
$$

which has the following estimate $\left\|\widetilde{u}_{\varepsilon}\right\|_{H^{1}\left(\Omega ; \mathbb{R}^{2}\right)}=o(\varepsilon)$. Moreover, the points $\zeta$ and $\xi$ in (77) belong to the interval $(x, \widehat{x})$.

By considering a polar system of coordinates $(r, \phi)$ centered at point $\widehat{x}$ (center of the inclusion $B_{\varepsilon}$ ) and aligned with the principal directions of the tensor $S(u, \theta)$ associated to the original domain $\Omega$, the components of the tensor $\sigma_{\varepsilon}\left(w_{\varepsilon}\right)$ are given by: 
- Exterior solution $(r \geq \varepsilon)$

$$
\begin{aligned}
\sigma_{\varepsilon}\left(w_{\varepsilon}\right)^{r r} & =-\frac{1-\gamma^{M}}{1+a \gamma^{M}} \frac{\varepsilon^{2}}{r^{2}}\left(\frac{\sigma_{1}+\sigma_{2}}{2}\right)-\frac{1-\gamma^{M}}{1+b \gamma^{M}} \frac{\varepsilon^{2}}{r^{2}}\left(4-3 \frac{\varepsilon^{2}}{r^{2}}\right)\left(\frac{\sigma_{1}-\sigma_{2}}{2}\right) \cos 2 \phi \\
& +\frac{1-\gamma^{M} \gamma^{C}}{1+a \gamma^{M}} \frac{\varepsilon^{2}}{r^{2}}\left(\frac{Q_{1}+Q_{2}}{2}\right), \\
\sigma_{\varepsilon}\left(w_{\varepsilon}\right)^{\phi \phi} & =\frac{1-\gamma^{M}}{1+a \gamma^{M}} \frac{\varepsilon^{2}}{r^{2}}\left(\frac{\sigma_{1}+\sigma_{2}}{2}\right)-3 \frac{1-\gamma^{M}}{1+b \gamma^{M}} \frac{\varepsilon^{4}}{r^{4}}\left(\frac{\sigma_{1}-\sigma_{2}}{2}\right) \cos 2 \phi \\
& -\frac{1-\gamma^{M} \gamma^{C}}{1+a \gamma^{M}} \frac{\varepsilon^{2}}{r^{2}}\left(\frac{Q_{1}+Q_{2}}{2}\right), \\
\sigma_{\varepsilon}\left(w_{\varepsilon}\right)^{\phi r} & =-\frac{1-\gamma^{M}}{1+b \gamma^{M}} \frac{\varepsilon^{2}}{r^{2}}\left(2-3 \frac{\varepsilon^{2}}{r^{2}}\right)\left(\frac{\sigma_{1}-\sigma_{2}}{2}\right) \sin 2 \phi .
\end{aligned}
$$

- Interior solution $(0<r<\varepsilon)$

$$
\begin{aligned}
\sigma_{\varepsilon}\left(w_{\varepsilon}\right)^{r r} & =\frac{a \gamma^{M}\left(1-\gamma^{M}\right)}{1+a \gamma^{M}}\left(\frac{\sigma_{1}+\sigma_{2}}{2}\right)+\frac{b \gamma^{M}\left(1-\gamma^{M}\right)}{1+b \gamma^{M}}\left(\frac{\sigma_{1}-\sigma_{2}}{2}\right) \cos 2 \phi \\
& -\frac{a \gamma^{M}\left(1-\gamma^{M} \gamma^{C}\right)}{1+a \gamma^{M}}\left(\frac{Q_{1}+Q_{2}}{2}\right), \\
\sigma_{\varepsilon}\left(w_{\varepsilon}\right)^{\phi \phi} & =\frac{a \gamma^{M}\left(1-\gamma^{M}\right)}{1+a \gamma^{M}}\left(\frac{\sigma_{1}+\sigma_{2}}{2}\right)-\frac{b \gamma^{M}\left(1-\gamma^{M}\right)}{1+b \gamma^{M}}\left(\frac{\sigma_{1}-\sigma_{2}}{2}\right) \cos 2 \phi \\
& -\frac{a \gamma^{M}\left(1-\gamma^{M} \gamma^{C}\right)}{1+a \gamma^{M}}\left(\frac{Q_{1}+Q_{2}}{2}\right), \\
\sigma_{\varepsilon}\left(w_{\varepsilon}\right)^{\phi r} & =-\frac{b \gamma^{M}\left(1-\gamma^{M}\right)}{1+b \gamma^{M}}\left(\frac{\sigma_{1}-\sigma_{2}}{2}\right) \sin 2 \phi .
\end{aligned}
$$

where $\sigma_{1,2}$ and $Q_{1,2}$ are, respectively, the principal stress associated to the tensor $\sigma(u)$ and $Q(\theta)$ of the unperturbed domain $\Omega$, evaluated at the point $\widehat{x} \in \Omega$. Moreover, the constants $a$ and $b$ in (78) to (83) depend only on Poisson's ratio $\nu$ of the matrix, and are given by

$$
a=\frac{1+\nu}{1-\nu} \quad \text { and } \quad b=\frac{3-\nu}{1+\nu} .
$$

Finally, using the asymptotic expansions presented in (78) to (83), we have that the derivative $\dot{\mathcal{J}}_{\chi_{\varepsilon}}$ is given by the following expression:

$$
\begin{aligned}
\dot{\mathcal{J}}_{\chi_{\varepsilon}}\left(u_{\varepsilon}, \theta\right)= & -\frac{\pi \varepsilon}{E}\left(\frac{1-\gamma^{M}}{1+b \gamma^{M}}\right)\left[4 \sigma(u) \cdot \sigma(u)+\frac{\gamma^{M}(b-2 a)-1}{1+a \gamma^{M}}(\operatorname{tr} \sigma(u))^{2}\right] \\
& -\frac{\pi \varepsilon}{2 E}\left(\frac{1-\gamma^{M} \gamma^{C}}{1+a \gamma^{M}}\right)\left[\left(1-\gamma^{M} \gamma^{C}\right)(1+\nu)(\operatorname{tr} Q(\theta))^{2}-4 \operatorname{tr} \sigma(u) \operatorname{tr} Q(\theta)\right] \\
& +o(\varepsilon)
\end{aligned}
$$

where $\operatorname{tr}(\cdot)$ denotes the trace operator of tensor $(\cdot)$. 
3.2. Case $\gamma^{M}=1$ and $\gamma^{C}=1$

In this case the restriction $\gamma^{M}=1$ and $\gamma^{C}=1$ is introduced in expression (44), then the derivative of the shape functional $\mathcal{J}_{\chi_{\varepsilon}}\left(u_{\varepsilon}, \theta_{\varepsilon}\right)$ is given by:

$$
\dot{\mathcal{J}}_{\chi_{\varepsilon}}\left(u_{\varepsilon}, \theta_{\varepsilon}\right)=\int_{\partial B_{\varepsilon}} \llbracket\left(q_{\varepsilon}\left(\theta_{\varepsilon}\right) \cdot \nabla \varphi_{\varepsilon}\right) I-2 q_{\varepsilon}\left(\theta_{\varepsilon}\right) \otimes_{s} \nabla \varphi_{\varepsilon} \rrbracket n \cdot n+\mathcal{E}(\varepsilon),
$$

where the term $\mathcal{E}(\varepsilon)$ is given by

$$
\mathcal{E}(\varepsilon)=-\int_{\Omega} Q_{\varepsilon}\left(\theta_{\varepsilon}^{\prime}\right) \cdot \nabla\left(u_{\varepsilon}-u\right)^{s} .
$$

The temperature field $\theta_{\varepsilon}$ associated to the perturbed problem admits the following asymptotic expansion:

$$
\theta_{\varepsilon}(x)=\theta(x)+v_{\varepsilon}(x / \varepsilon)+\widetilde{\theta}_{\varepsilon}(x),
$$

where $v_{\varepsilon}$ is the solution of the exterior problem

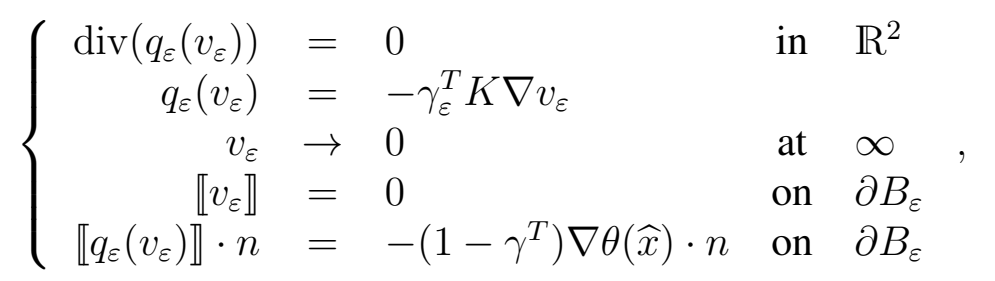

and the residue $\widetilde{\theta}_{\varepsilon}$ must be satisfies the following equation:

$$
\left\{\begin{aligned}
\operatorname{div}\left(q_{\varepsilon}\left(\widetilde{\theta}_{\varepsilon}\right)\right) & =0 & & \\
q_{\varepsilon}\left(\widetilde{\theta}_{\varepsilon}\right) & =-\gamma_{\varepsilon}^{T} K \nabla \widetilde{\theta}_{\varepsilon} & & \text { in } \Omega \\
\widetilde{\theta}_{\varepsilon} & =-v_{\varepsilon} & & \text { on } \Gamma_{D_{T}} \\
q_{\varepsilon}\left(\widetilde{\theta}_{\varepsilon}\right) \cdot n & =-q_{\varepsilon}\left(v_{\varepsilon}\right) \cdot n & & \text { on } \Gamma_{N_{T}} \\
\llbracket q_{\varepsilon}\left(\widetilde{\theta}_{\varepsilon}\right) \rrbracket \cdot n & =\varepsilon\left(1-\gamma^{T}\right)(\nabla q(\theta(\vartheta)) n) \cdot n & & \text { on } \partial B_{\varepsilon}
\end{aligned}\right.
$$

which has the following estimate $\left\|\widetilde{\theta}_{\varepsilon}\right\|_{H^{1}(\Omega)}=o(\varepsilon)$. Moreover, the point $\vartheta$ in (90) belongs to the interval $(x, \widehat{x})$. In addition, the solution $v_{\varepsilon}$ to the exterior problem can be explicitly written as:

- Exterior solution $(r \geq \varepsilon)$

$$
v_{\varepsilon}(x / \varepsilon)=\frac{1-\gamma^{T}}{1+\gamma^{T}} \frac{\varepsilon^{2}}{\|x-\widehat{x}\|^{2}} \nabla \theta(\widehat{x}) \cdot(x-\widehat{x}) .
$$

- Interior solution $(0<r<\varepsilon)$

$$
v_{\varepsilon}(x / \varepsilon)=\frac{1-\gamma^{T}}{1+\gamma^{T}} \nabla \theta(\widehat{x}) \cdot(x-\widehat{x}) .
$$

On the other hand, the field $\varphi_{\varepsilon}$ - solution of the auxiliary problem (30) - admits the following asymptotic expansion

$$
\varphi_{\varepsilon}(x)=\varphi(x)+p_{\varepsilon}(x / \varepsilon)+\widetilde{\varphi}_{\varepsilon}(x),
$$


where $p_{\varepsilon}$ is the solution of the exterior problem

$$
\left\{\begin{aligned}
\operatorname{div}\left(q_{\varepsilon}\left(p_{\varepsilon}\right)\right) & =0 & & \text { in } \mathbb{R}^{2} \\
q_{\varepsilon}\left(p_{\varepsilon}\right) & =-\gamma_{\varepsilon}^{T} K \nabla p_{\varepsilon} & & \\
p_{\varepsilon} & \rightarrow 0 & & \text { at } \\
\llbracket p_{\varepsilon} \rrbracket & =0 & & \text { on } \partial B_{\varepsilon} \\
\llbracket q_{\varepsilon}\left(p_{\varepsilon}\right) \rrbracket \cdot n & =-\left(1-\gamma^{T}\right) \nabla \varphi(\widehat{x}) \cdot n & & \text { on } \partial B_{\varepsilon}
\end{aligned}\right.
$$

and the residue $\widetilde{\varphi}_{\varepsilon}$ must be satisfies the following equation:

$$
\left\{\begin{array}{rlrl}
\operatorname{div}\left(q_{\varepsilon}\left(\widetilde{\varphi}_{\varepsilon}\right)\right) & =0 & & \text { in } \Omega \backslash \overline{B_{\varepsilon}} \\
\operatorname{div}\left(q_{\varepsilon}\left(\widetilde{\varphi}_{\varepsilon}\right)\right) & =-\left(1-\gamma^{T}\right) \mathbb{C} B \cdot \nabla u^{s} & & \text { in } B_{\varepsilon} \\
q_{\varepsilon}\left(\widetilde{\varphi}_{\varepsilon}\right) & =-\gamma_{\varepsilon}^{T} K \nabla \widetilde{\varphi}_{\varepsilon} & & \\
\widetilde{\varphi}_{\varepsilon} & =-p_{\varepsilon} & & \text { on } \Gamma_{D_{T}} \\
q_{\varepsilon}\left(\widetilde{\varphi}_{\varepsilon}\right) \cdot n & =-q_{\varepsilon}\left(p_{\varepsilon}\right) & \text { on } \Gamma_{N_{T}} \\
\llbracket q_{\varepsilon}\left(\widetilde{\varphi}_{\varepsilon}\right) \rrbracket \cdot n & =\varepsilon\left(1-\gamma^{T}\right)(\nabla q(\varphi(\varrho)) n) \cdot n & & \text { on } \partial B_{\varepsilon}
\end{array},\right.
$$

which has the following estimate $\left\|\widetilde{\varphi}_{\varepsilon}\right\|_{H^{1}(\Omega)}=o(\varepsilon)$. Moreover, the point $\varrho$ in (95) belongs to the interval $(x, \widehat{x})$. In addition, the solution $\varphi_{\varepsilon}$ to the exterior problem can be explicitly written as:

- Exterior solution $(r \geq \varepsilon)$

$$
p_{\varepsilon}(x / \varepsilon)=\frac{1-\gamma^{T}}{1+\gamma^{T}} \frac{\varepsilon^{2}}{\|x-\widehat{x}\|^{2}} \nabla \varphi(\widehat{x}) \cdot(x-\widehat{x}) .
$$

- Interior solution $(0<r<\varepsilon)$

$$
p_{\varepsilon}(x / \varepsilon)=\frac{1-\gamma^{T}}{1+\gamma^{T}} \nabla \varphi(\widehat{x}) \cdot(x-\widehat{x}) .
$$

Finally, using the asymptotic expansions presented in (91), (92), (96) and (97), and recalling the estimate for (87); we have that the derivative of the functional $\mathcal{J}_{\chi_{\varepsilon}}\left(u_{\varepsilon}, \theta_{\varepsilon}\right)$ is given by the following expression:

$$
\dot{\mathcal{J}}_{\chi_{\varepsilon}}\left(u_{\varepsilon}, \theta_{\varepsilon}\right)=-4 \pi \varepsilon \frac{1-\gamma^{T}}{1+\gamma^{T}} \nabla \theta \cdot \nabla \varphi+o(\varepsilon)
$$

\subsection{Topological Derivative}

In order to calculate the topological derivative, we shall adopt the methodology developed in [17], whereby the topological derivative is obtained as

$$
D_{T}(\widehat{x})=\lim _{\varepsilon \rightarrow 0} \frac{1}{f^{\prime}(\varepsilon)} \dot{\mathcal{J}}_{\chi_{\varepsilon}}\left(u_{\varepsilon}, \theta_{\varepsilon}\right)
$$

where the function $f(\varepsilon)$ is the size of the perturbation, i.e. $f(\varepsilon)=\pi \varepsilon^{2} \Rightarrow f^{\prime}(\varepsilon)=2 \pi \varepsilon$.

Due to the linearity property of the shape functional with respect to the thermal problem (26), it is possible to write the topological derivative of the functional $\mathcal{J}_{\chi_{\varepsilon}}\left(u_{\varepsilon}, \theta_{\varepsilon}\right)$ based 
on the results given in (85) and (98). Then, the final expression of the topological derivative becomes a scalar function defined over the unperturbed domain $\Omega$, that is

$$
\begin{aligned}
D_{T}(\widehat{x})= & -\frac{1}{2 E}\left(\frac{1-\gamma^{M}}{1+b \gamma^{M}}\right)\left[4 \sigma(u) \cdot \sigma(u)+\frac{\gamma^{M}(b-2 a)-1}{1+a \gamma^{M}}(\operatorname{tr} \sigma(u))^{2}\right] \\
& -\frac{1}{4 E}\left(\frac{1-\gamma^{M} \gamma^{C}}{1+a \gamma^{M}}\right)\left[\left(1-\gamma^{M} \gamma^{C}\right)(1+\nu)(\operatorname{tr} Q(\theta))^{2}-4 \operatorname{tr} \sigma(u) \operatorname{tr} Q(\theta)\right] \\
& -2 \frac{1-\gamma^{T}}{1+\gamma^{T}} \nabla \theta \cdot \nabla \varphi .
\end{aligned}
$$

Notice that the first term is classic in the topological asymptotic analysis for the elasticity problem. The linearity property mentioned previously appears explicitly in the last term of the above results, see term involving the contrast parameter $\gamma^{T}$. On the other hand, the nonlinear dependence of the problem with the thermo-elastic constitutive properties appears, also explicitly, in the term with the contrast parameters $\gamma^{M} \gamma^{C}$. These two last terms represents the contribution of the thermal problem to the elastic stress problem.

For study the sensitivity of the problem to the insertion of a hole in the domain, we can take the limit of the (100) when the contrasts $\gamma^{M}, \gamma^{C}$ and $\gamma^{T}$ tend simultaneously to 0 . Then, for this particular case, the topological derivative as the form

$\left.D_{T}(\widehat{x})\right|_{\text {hole }}=-\frac{1}{2 E}\left[4 \sigma(u) \cdot \sigma(u)-(\operatorname{tr} \sigma(u))^{2}\right]-\frac{\operatorname{tr} Q(\theta)}{4 E}[(1+\nu) \operatorname{tr} Q(\theta)-4 \operatorname{tr} \sigma(u)]-2 \nabla \theta \cdot \nabla \varphi$.

\section{Final comments}

The topological derivative in its closed form for the total potential mechanical energy associated to a thermo-mechanical semi-coupled system, when a circular inclusion is introduced at an arbitrary point of the domain, has been derived. In particular, the linear elasticity system (modeled by the Navier equation) coupled with the steady-state heat conduction problem (modeled by the Laplace equation) has been considered. The mechanical coupling term comes out from the thermal stress induced by the temperature field. Since this term is nonlocal, a non-standard adjoint state has been introduced, which allowed to obtain a closed form for the topological derivative. In addition, a full mathematical justification for the derived formulas and precise estimates for the remainders of the topological asymptotic expansion have been provided. Finally, we remark that this information can be potentially used in a number of applications of practical interest such as multi-physic topology design of structures under mechanical and/or thermal loads.

\section{References}

[1] G. Allaire, F. Jouve, and N. Van Goethem. Damage and fracture evolution in brittle materials by shape optimization methods. Journal of Computational Physics, 230:50105044, 2011.

[2] S. Amstutz. Sensitivity analysis with respect to a local perturbation of the material property. Asymptotic Analysis, 49(1-2):87-108, 2006. 
[3] S. Amstutz, S.M. Giusti, A.A. Novotny, and E.A. de Souza Neto. Topological derivative in multi-scale linear elasticity models applied to the synthesis of microstructures. International Journal for Numerical Methods in Engineering, 84(6):733-756, 2010.

[4] S. Amstutz, I. Horchani, and M. Masmoudi. Crack detection by the topological gradient method. Control and Cybernetics, 34(1):81-101, 2005.

[5] S. Amstutz and A.A. Novotny. Topological optimization of structures subject to von mises stress constraints. Structural and Multidisciplinary Optimization, 41(3):407-420, 2010.

[6] M.C. Delfour and J.P. Zolésio. Shapes and Geometries. Advances in Design and Control, vol. 4. SIAM, Philadelphia, 2001.

[7] H.A. Eschenauer, V.V. Kobelev, and A. Schumacher. Bubble method for topology and shape optmization of structures. Structural Optimization, 8(1):42-51, 1994.

[8] J.D. Eshelby. The elastic energy-momentum tensor. Journal of Elasticity, 5(3-4):321$335,1975$.

[9] S.M. Giusti, A.A. Novotny, E.A. de Souza Neto, and R.A. Feijóo. Sensitivity of the macroscopic elasticity tensor to topological microstructural changes. Journal of the Mechanics and Physics of Solids, 57(3):555-570, 2009.

[10] N. Van Goethem and A.A. Novotny. Crack nucleation sensitivity analysis. Mathematical Methods in the Applied Sciences, 33(16):1978-1994, 2010.

[11] M.E. Gurtin. An introduction to continuum mechanics. Mathematics in Science and Engineering vol. 158. Academic Press, New York, 1981.

[12] M.E. Gurtin. Configurational forces as basic concept of continuum physics. Applied Mathematical Sciences vol. 137. Springer-Verlag, New York, 2000.

[13] M. Hintermüller and A. Laurain. Multiphase image segmentation and modulation recovery based on shape and topological sensitivity. Journal on Mathematical Imaging and Vision, 35:1-22, 2009.

[14] M. Hintermüller, A. Laurain, and A.A. Novotny. Second-order topological expansion for electrical impedance tomography. Advances in Computational Mathematics, 36(2):235$265,2012$.

[15] I. Larrabide, R.A. Feijóo, A.A. Novotny, and E. Taroco. Topological derivative: a tool for image processing. Computers \& Structures, 86(13-14):1386-1403, 2008.

[16] S.A. Nazarov and J. Sokołowski. Asymptotic analysis of shape functionals. Journal de Mathématiques Pures et Appliquées, 82(2):125-196, 2003.

[17] A.A. Novotny. Análise de sensibilidade topológica. Phd thesis, Laboratório Nacional de Computação Científica, Petrópolis, Brazil, 2003. 
[18] A.A. Novotny, R.A. Feijóo, E. Taroco, and C. Padra. Topological sensitivity analysis for three-dimensional linear elasticity problem. Computer Methods in Applied Mechanics and Engineering, 196(41-44):4354-4364, 2007.

[19] J. Sokołowski and A. Żochowski. On the topological derivative in shape optimization. SIAM Journal on Control and Optimization, 37(4):1251-1272, 1999.

[20] J. Sokołowski and J.P. Zolésio. Introduction to shape optimization - shape sensitivity analysis. Springer Series in Computational Mathematics, vol. 16. Springer-Verlag, Berlin, 1992. 